\title{
Diagnosis of post-transplant coronary artery disease using contrast-enhanced coronary vessel wall imaging at 3.0 Tesla
}

\author{
Tarique Hussain ${ }^{1 *}$, Matthew Fenton ${ }^{2}$, Sarah A Peel ${ }^{1}$, Andrea Wiethoff ${ }^{1,3}$, Rene M Botnar ${ }^{1}$, Michael Burch², \\ Gerald F Greil ${ }^{1}$
}

From 15th Annual SCMR Scientific Sessions

Orlando, FL, USA. 2-5 February 2012

\section{Summary}

Coronary plaque characterization at 3.0 Tesla holds great potential for clinical benefit. Coronary allograft vasculopathy (CAV) occurs after heart transplantation and is an immune-mediated diffuse coronary intimal disease. We demonstrate the use of contrast-enhanced inversion-recovery prepared coronary vessel wall MRI at $3 \mathrm{~T}$ for diagnosis. We further validate the technique in 23 patients with intravascular ultrasound (IVUS), showing an accuracy of $91 \%$. Direct non-invasive imaging has great potential benefit for screening and prevention in these patients. The study has further significant implications for conventional coronary atherosclerosis.

\section{Background}

Coronary Allograft Vasculopathy (CAV) remains the leading cause of late death after heart transplantation in children but is poorly detected by X-ray angiography. Intravascular ultrasound (IVUS) is invasive and costly, which precludes close follow-up. We have previously demonstrated the feasibility of MRI late gadolinium enhancement (LGE) in the coronary vessel wall to detect and grade CAV. The purpose of this study was to demonstrate the clinical utility of the technique and if it could be improved by imaging at 3.0 Tesla.

\section{Methods}

Participants underwent IVUS and cardiac MRI. Maximal intimal thickness (MIT), mean intimal index (MII = mean intimal area/ mean vessel area) and Stanford grade (0 to 4 (severe)) were recorded on IVUS. MRI

'Division of Imaging Sciences, King's College London, London, UK Full list of author information is available at the end of the article included a coronary magnetic resonance angiogram (CMRA) of the left coronary artery and LGE vessel wall imaging after administration of $0.2 \mathrm{mmol} / \mathrm{kg}$ Gadolinium. Participants were randomised to $3 \mathrm{~T}$ or $1.5 \mathrm{~T}$ (Achieva, Philips Healthcare, Best, Netherlands). 32-element cardiac phased-array receiver coils were used. The mean enhancement diameter (ED) and index $(\mathrm{Ei}=$ mean enhancement area/ mean vessel area on MRI) was quantified on LGE images fused with CMRA as a roadmap. Additionally, images were scored for inter/intra-observer error in analysis and image quality (0 (not seen) to 4 (sharp image)). Location of LGE on MRI and location of significant intimal thickening on IVUS were also recorded for comparison.

\section{Results}

24 adolescents participated in the study (characteristics: Table 1). One MRI failed due to patient discomfort. Overall, there was excellent correlation of MRI with IVUS. Pearson's correlation for ED with MIT was 0.80 $(\mathrm{p}<0.001)$ and for Ei with MII was $0.92(\mathrm{p}<0.001)$. Correlation coefficients at $3 \mathrm{~T}$ were similar to $1.5 \mathrm{~T}$ ( 0.77 \& 0.96 versus $0.81 \& 0.62$ respectively (all $\mathrm{p}<0.05)$ ). BlandAltman analysis also shows similar inter/intra-observer agreement between the two field strengths.

However, LGE image quality was improved at $3 \mathrm{~T}$ $($ median $=3$ ) compared to $1.5 \mathrm{~T}$ (median $=2 ; \mathrm{p}=0.019$ by Mann-Whitney test). Furthermore, at $1.5 \mathrm{~T}$ only 11 out of 17 areas of enhancement on MRI were associated with corresponding significant disease on IVUS (i.e. location match to within $1 \mathrm{~mm}$ from a major branchpoint). At 3T, however, 11 of 13 enhancing lesions had exact and anatomical match (Figure 1). 


\section{Table 1 Patient Characteristics}

\begin{tabular}{ccc}
\hline & 1.5 TESLA & 3.0 TESLA \\
\hline Number of Patients & 12 & 11 \\
Male $(\mathrm{n}=)$ & 5 & 5 \\
Age (years) & $15.1 \pm 2.2$ years & $15.8 \pm 1.7$ years \\
Weight $(\mathrm{kg})$ & $58.9 \pm 20.9$ & $57.7 \pm 10.9$ \\
Height $(\mathrm{cm})$ & $162 \pm 9$ & $164 \pm 11$ \\
HR (bpm) & $81 \pm 8$ & $88 \pm 8$ \\
MIT (mm) & $0.50 \pm 0.29$ & $0.98 \pm 0.56$ \\
MII (\%) & $17.5 \pm 5.7$ & $24.7 \pm 13.5$ \\
ED (mm) & $5.38 \pm 2.39$ & $7.86 \pm 6.57$ \\
Ei & $0.53 \pm 0.42$ & $1.72 \pm 2.35$ \\
Stanford Grade 4 disease $(\mathrm{n}=)$ & 2 & 5 \\
Previous CMV infection $(\mathrm{n}=)$ & 2 & 1 \\
Hypertension ( $\mathrm{n}=)$ & 10 & 7 \\
Donor Age (years) & $23.3 \pm 11.9$ & $25.1 \pm 16.1$ \\
\hline
\end{tabular}

Overall, the receiver operating characteristics curve demonstrates that a cut-off of $7.5 \mathrm{~mm}$ ED on MRI has $86 \%$ sensitivity and $94 \%$ specificity for the detection of significant (Stanford grade 4 on IVUS) CAV. This gives a positive predictive value of $86 \%$, negative predictive value of $94 \%$ and accuracy of $91 \%$.

In the multivariate analysis, donor age, length of time post transplant and MRI (ED) were the only significant independent predictors of maximum intimal thickness on IVUS.

\section{Conclusions}

Coronary vessel wall delayed enhancement MRI is a valuable and accurate non-invasive method to quantify CAV. Improved image quality at 3.0 Tesla further ameliorates the accuracy of this technique.

Where appropriate, mean \pm standard deviation is given.



Figure 1 A) Patient 1 Left Coronary system CMRA at 3T. B) Patient 1 LGE at 3T. C) Patient 2 Left Coronary CMRA at 1.5T. D) Patient 2 LGE at 1.5T E) Overlay of Patient 1 LGE on CMRA at 3T. Arrows show corresponding positions for IVUS pictures G \& H. F) Overlay of Patient 2 LGE on CMRA at 1.5T. Arrows show corresponding positions for IVUS pictures I \& J. G \& I) IVUS images. White box illustrates intimal thickening corresponding to enhancement on overlay picture above J \& H) IVUS images. No significant intimal thickening corresponding to areas without enhancement on overlay picture above 


\section{Funding}

Funding: We would like to thank the Well Child Foundation (Cheltenham, UK) for financial support of the Senior Clinical Lecturer position of Gerald F. Greil, MD. The authors acknowledge financial support from the Department of Health via the National Institute for Health Research (NIHR) comprehensive Biomedical Research Centre award to Guy's \& St Thomas' NHS Foundation Trust in partnership with King's College London and King's College Hospital NHS Foundation Trust. The MRI scanner is partly supported by Philips Healthcare. A. Wiethoff is an employee of Philips Healthcare, Best. All the other authors were not consultants or employees for Philips Healthcare and had control of inclusion of any data and information that might present a conflict of interest for A. Wiethoff.

No financial support was provided for data collection and analysis or manuscript preparation.

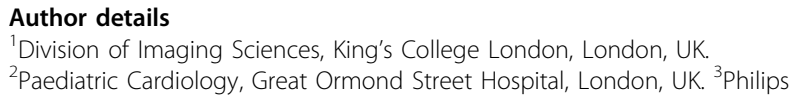

Published: 1 February 2012 